\title{
Force feedback effects on single molecule hopping and pulling experiments
}

\author{
M. Rico-Pasto, ${ }^{1}$ I. Pastor, ${ }^{1,2}$ and F. Ritort ${ }^{1,2}$ \\ ${ }^{1}$ Departament de Fisica de la Materia Condensada, Universitat de Barcelona, C/ Marti i Franques 1, \\ O8028 Barcelona, Spain \\ ${ }^{2}$ CIBER_BNN, Instituto de Salud Carlos III, 28029 Madrid, Spain
}

(Received 24 October 2017; accepted 15 January 2018; published online 1 February 2018)

\begin{abstract}
Single-molecule experiments with optical tweezers have become an important tool to study the properties and mechanisms of biological systems, such as cells and nucleic acids. In particular, force unzipping experiments have been used to extract the thermodynamics and kinetics of folding and unfolding reactions. In hopping experiments, a molecule executes transitions between the unfolded and folded states at a preset value of the force [constant force mode (CFM) under force feedback] or trap position [passive mode (PM) without feedback] and the force-dependent kinetic rates extracted from the lifetime of each state (CFM) and the rupture force distributions (PM) using the Bell-Evans model. However, hopping experiments in the CFM are known to overestimate molecular distances and folding free energies for fast transitions compared to the response time of the feedback. In contrast, kinetic rate measurements from pulling experiments have been mostly done in the PM while the CFM is seldom implemented in pulling protocols. Here, we carry out hopping and pulling experiments in a short DNA hairpin in the PM and CFM at three different temperatures $\left(6{ }^{\circ} \mathrm{C}, 25{ }^{\circ} \mathrm{C}\right.$, and $\left.45^{\circ} \mathrm{C}\right)$ exhibiting largely varying kinetic rates. As expected, we find that equilibrium hopping experiments in the CFM and PM perform well at $6{ }^{\circ} \mathrm{C}$ (where kinetics are slow), whereas the CFM overestimates molecular parameters at $45{ }^{\circ} \mathrm{C}$ (where kinetics are fast). In contrast, nonequilibrium pulling experiments perform well in both modes at all temperatures. This demonstrates that the same kind of feedback algorithm in the CFM leads to more reliable determination of the folding reaction parameters in irreversible pulling experiments. Published by AIP Publishing. https://doi.org/10.1063/1.5010303
\end{abstract}

\section{INTRODUCTION}

The invention of single-molecule manipulation techniques over the past decades has provided new insights into the details of complex molecular reactions in cells ${ }^{1-5}$ that complement traditional bulk methods. Techniques such as optical tweezers ${ }^{6,7}$ allow scientists to mechanically unzip and stretch single biomolecules like DNA, ${ }^{8-10} \mathrm{RNA}^{11,12}$ and proteins $^{13-15}$ in a controlled manner.

In DNA unzipping experiments, a tensile force is applied to the $3^{\prime}$ and $5^{\prime}$ of a DNA hairpin until the base pairs (bp's) that stabilize the double helix are disrupted and the native hairpin unfolds and is converted into single-stranded DNA (ssDNA). ${ }^{10,16,17}$ The unzipping experiment is the equivalent of a temperature-induced melting process, the main difference being the final state of the DNA hairpin: a stretched polymer in the former versus a random coil in the latter. The reverse of the unzipping process is molecular folding or zipping: upon releasing the force, the stretched ssDNA folds back into the native hairpin in a process determined by the nucleation of the loop and the formation of the stem. The thermodynamics and kinetics of the unzipping-zipping reaction give valuable information about the free energy of folding of the DNA hairpin and the underlying molecular free energy landscape otherwise difficult to obtain in bulk assays. The unfolding and folding reaction can be studied in two types of force spectroscopy protocols using optical tweezers: hopping and pulling. Moreover, experiments can be carried out in two control modes: constant-force mode (CFM) and passive mode (PM) depending on whether the force (CFM) or the trap position (PM) is the control parameter (i.e., the externally controlled variable that can be held fixed and is not subject to thermal fluctuations).

In hopping experiments, the control parameter (trap position or force) is kept constant while the molecule executes transitions between the folded and unfolded states. ${ }^{18-21}$ In contrast, in pulling experiments, the control parameter is repeatedly and continuously changed between two limit values while the force-distance curve is recorded along the stretching-releasing cycles. ${ }^{22,23}$ Single-molecule experiments monitor transitions in real time, allowing us to measure transiently fast and rare events that would otherwise be masked in bulk assays where only averages over a large number of molecules are measured.

The force-dependence of the measured unfolding and folding kinetic rates is typically studied using the Bell-Evans (BE) model ${ }^{24,25}$ where the unfolding-folding process is a thermally activated diffusive process that passes through a transition state (TS) characterized by a kinetic barrier. Kinetic rates exhibit an Arrhenius-like exponential dependence where the kinetic barrier in the exponent changes linearly with force. Fitting the BE model to the experimentally measured kinetic rates allows us to estimate the distance from each state (folded and unfolded) to the transition state. ${ }^{20,26}$ The BE model is based on the force ensemble, i.e., force is the control parameter in the experiment. In an optical tweezer setup, 
however, the position of the center of the optical trap rather than force is the natural control parameter. Because kinetics is directly dependent on force, scientists have made efforts to implement algorithms of force feedback to control directly the force in hopping experiments. ${ }^{18,19}$ However, force feedback algorithms present artifacts due to their finite bandwidth that leads to limited finite response time of the device and additional low frequency colored noise acting upon the system. The first artifact leads to fast transitions being missed during the algorithm feedback cycle and an overestimation of the molecular extension is obtained from kinetic rate measurements. ${ }^{19}$

In this work, we have investigated the kinetics of unfolding and folding for a DNA hairpin at different temperatures using the PM and CFM in hopping and pulling experiments. Experiments using optical trap setups with CFM have been conducted in the past to stretch biopolymers at a given force and to monitor hopping transitions at a given force in molecular folders such as nucleic acid hairpins and proteins. ${ }^{2,11,18,19,27,28}$ Here we present a new implementation of this kind of protocol by pulling a DNA hairpin in a ramping force experiment where force is controlled in real time by the feedback loop. To the best of our knowledge, this kind of pulling protocol has never been implemented in optical tweezers. It must be said, though, that in other setups such as magnetic and acoustic tweezers, the CFM remains as the natural pulling protocol. We carried out hopping experiments using the $\mathrm{PM}$ and $\mathrm{CFM}$ at three different temperatures over a broad range $\left(6^{\circ} \mathrm{C}, 25^{\circ} \mathrm{C}\right.$, and $\left.45^{\circ} \mathrm{C}\right)$ using a miniaturized optical tweezer setup which allows us to measure the exerted force to the molecule. The instrument operates in a cold mode by cooling the medium using an icebox and in a hot mode by heating the medium from room temperature using a collimated heating laser (see details in Ref. 7). Controlling temperature enables us to explore folding and unfolding kinetics of single molecules over a wide range of time scales due to the strong dependence of kinetic rates on temperature. The large change in kinetic rates makes then possible to assess the effect of missed transitions on kinetics measurements in the CFM. This has been done in Refs. 18 and 19 for hopping transitions at room temperature $\left(25^{\circ} \mathrm{C}\right)$ and here it is extended to low and high temperatures and for pulling experiments too. Experiments in the PM were done at the same three temperatures as for the CFM to cross-check the validity of the CFM results.

\section{MATERIALS AND METHODS}

\section{A. Preparation of single-molecule construct}

The molecular construction has been designed as described in Ref. 18. It was synthesized using the hybridization of two different oligonucleotides. One contains the designed 20 base pair (bp) DNA hairpin (5'-GCGAGCCATAAT CTCATCTG GAAA CAGATGAGATTATGGCTCGC-3') flanked with two identical single-stranded DNA (ssDNA) handles. The other one has the complementary sequence of the DNA handles. After the ligation step, we have the hairpin between both 29 bp double-stranded DNA (dsDNA) handles. The molecular construction is tagged at one end with a single biotin and a multiple digoxigenin at the other end.

\section{B. Instrument design}

The instrument used in this study is a miniaturized counterpropagating optical tweezer setup (see details in Refs. 6 and 10) focused in a microfluidics chamber to hold a microscopic dielectric bead attached to one end of the molecular construction. The other end is fixed in the tip of a micro-pipette by air suction [Fig. 1(a)]. One bead is coated with streptavidin, whereas the other is coated with anti-dig. Connections to beads with the molecular construction are made through biotinstreptavidin and antigen-antibody bonds, respectively. The distance between the center of the optical trap and the immobilized bead is called $\lambda$ and is the natural control parameter of this device. The instrument can operate in two temperaturecontrolled modes: hot and cold. In the hot mode, a collimated laser of wavelength $1435 \mathrm{~nm}$ is placed coaxially to the trapping beams to heat the medium close to the trap. ${ }^{7}$ By tuning the laser power of this heating laser, it is possible to increase the temperature from room temperature $\left(25^{\circ} \mathrm{C}\right)$ to a maximum of $50{ }^{\circ} \mathrm{C}$ (hot mode). To operate in the cold mode, we introduce the head of the miniaturized tweezer setup inside a turned off icebox containing $20 \mathrm{~L}$ of ice to cold down the temperature and keep it constant $\left(4-5^{\circ} \mathrm{C}\right)$. By turning on the heating laser inside the icebox, it is possible to increase the temperature from $5^{\circ} \mathrm{C}$ to $30{ }^{\circ} \mathrm{C}$ (cold mode). Overall, the combined hot and cold modes give an operational temperature range between $5{ }^{\circ} \mathrm{C}$ and $50{ }^{\circ} \mathrm{C}$.

To determine the real temperature of the sample at each laser power, it is necessary to calibrate it using Stokes' Law
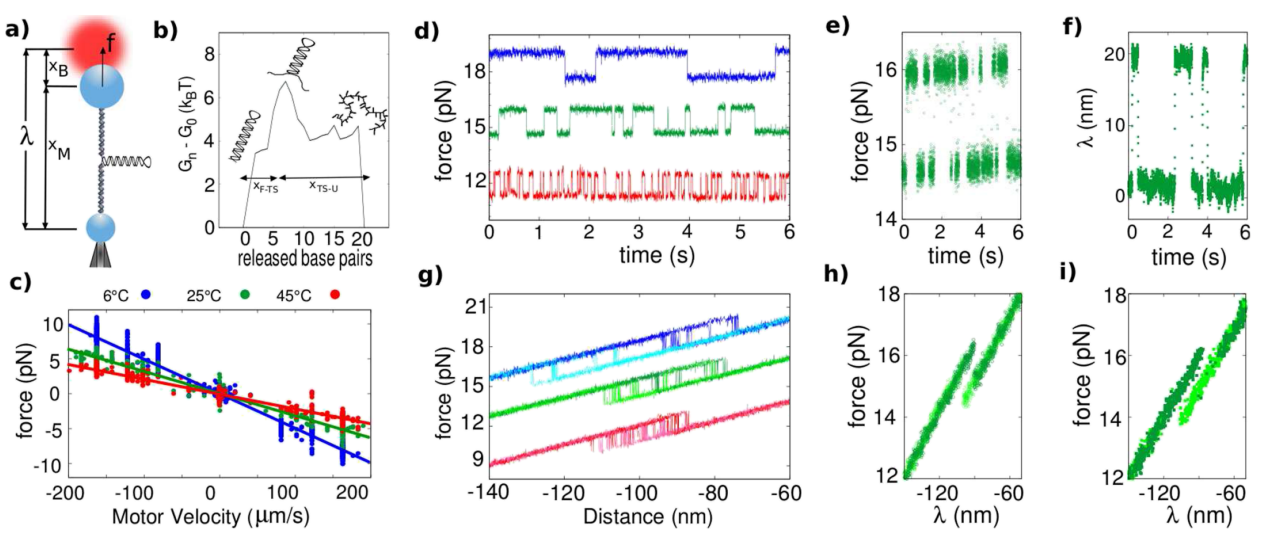

FIG. 1. (a) Schematic depiction of the experimental optical tweezer setup to manipulate a single molecule. (b) Energy landscape of the studied molecule at the coexistence force and room temperature. (c) Force versus velocity curve at three different heating powers. Blue points correspond to $6^{\circ} \mathrm{C}$, green points correspond to $25^{\circ} \mathrm{C}$, and red ones correspond to $45^{\circ} \mathrm{C}$. Hopping (d) and pulling (g) experiments in PM have the same color code as in (c). Hopping experiments in PM (e) and CFM (f) at $25^{\circ} \mathrm{C}$. Pulling experiments in PM (h) and $\mathrm{CFM}$ (i) at $25^{\circ} \mathrm{C}$. 
test at different trap powers [Fig. 1(c)]. By moving the bead hold in the optical trap at different velocities and recording the force, we can estimate changes in the viscosity of the medium from the measured slope in the force-velocity curves. Fitting the measured viscosity to the empirical Vogel equation, it is possible to determine the real temperature near the optical trap for each laser power.

\section{Hopping experiments}

In hopping experiments, the control parameter, $\lambda(\mathrm{PM})$ or force (CFM), is kept constant while folding and unfolding transitions are monitored [Figs. 1(d)-1(f)]. In the PM, the force jumps at each transition, the value of the jump being proportional to the number of released (unfolding) or retracted (folding) base pairs [Figs. 1(d) and 1(e)]. Also, it is possible to see in Fig. 1(d) how kinetics changes as a function of temperature: the higher the temperature, the faster the hopping kinetics. In the CFM force feedback acts to keep constant the force. When an unfolding or folding event takes place, the linear feedback algorithm calculates the trap displacement necessary to recover the preset force value [Fig. 1(f)]. From these experiments, we can recover directly the molecular extension at different forces. For both type of hopping experiments, CFM and PM, a partition method based on Gaussian fitting is used to estimate the lifetime of each state. The corresponding kinetic rate is defined as the inverse of the average lifetime. In the case of the CFM experiments, the relationship between the force and the kinetic rates is obtained directly from the data. In the PM, the force corresponding to the unfolding (folding) transition rates is defined as the average force measured in the folded (unfolded) state.

\section{Pulling experiments}

In pulling experiments, the control parameter is repeatedly moved back and forth between a minimum value corresponding to a low force where the molecule is folded to a maximal value corresponding to a large force [Figs. 1(g)-1(i)]. When the molecule unfolds (refolds) at a certain value of the control parameter, a jump is observed in the force signal. In pulling experiments in the CFM, when the molecule unfolds (refolds), the feedback transiently increases the pulling speed to recover the same force before the rip (jump). Changes in the trap distance are observed when the molecule changes its configuration [Fig. 1(i)]. Although rips in extension in pulling experiments in the CFM are expected, these are not clearly observed along the force-distance curve due to the limited bandwidth of the feedback that is not sufficiently rapid to follow the transitions.

In order to determine the kinetic rates involved in the melting process, we measure the first rupture and recovery forces along the unfolding and folding trajectories, respectively. From the histograms of such forces, we can estimate the survival probabilities for the folded and unfolded state. Finally, from the survival probabilities, it is possible to calculate the unfolding and folding kinetic rates by dividing the probability by the corresponding force histogram and multiplying it with the experimental loading rate (see Ref. 26 for details).

\section{E. Bell-Evans (BE) model}

Our molecular construct consists of a 20 bp DNA hairpin inserted between two identical short dsDNA handles of $29 \mathrm{bp}$. The sequence of the stem of the hairpin is designed for the hairpin to fold and unfold as a two-state system [energy landscape in Fig. 1(b)]. The molecular free energy landscape can then be modeled as a two-state system separated by a kinetic barrier. The unfolding/folding reaction rates of this hairpin can be schematically described by a unimolecular reaction pathway,

$$
\begin{aligned}
& k_{F \rightarrow U} \\
& F \rightleftarrows U, \\
& k_{F \leftarrow U}
\end{aligned}
$$

where $\mathrm{F}$ and $\mathrm{U}$ indicate the folded and unfolded states and $\mathrm{k}_{\mathrm{F} \rightarrow \mathrm{U}}$ and $\mathrm{k}_{\mathrm{F} \leftarrow \mathrm{U}}$ denote the force-dependent unfolding and folding rates, respectively.

The free energy landscape shows coexistence between both states close to $15 \mathrm{pN}$ and a ssDNA of released extension around $18 \mathrm{~nm}$ using the unified oligonucleotide or unzipping thermodynamic dataset and the elastic response of ssDNA at $25{ }^{\circ} \mathrm{C} .{ }^{10,20}$ The kinetic rates can be studied using the BE model,

$$
\begin{aligned}
& k_{F \rightarrow U}(f)=k_{0} \exp \left[\beta f x_{F-T S}\right], \\
& k_{F \leftarrow U}(f)=k_{0} \exp \left[\beta\left(\Delta G-f x_{T S-U}\right)\right],
\end{aligned}
$$

where $k_{0}$ is the kinetic attempt frequency of the molecule at zero force; $x_{F-T S}$ is the distance from $\mathrm{F}$ to the transition state (TS); $x_{T S-U}$ is the distance from TS to $\mathrm{U} ; \Delta G$ is the energy difference between $\mathrm{F}$ and $\mathrm{U}$; and $\beta=1 / \mathrm{k}_{\mathrm{B}} \mathrm{T}$ is the inverse of the product of the Boltzmann constant $\left(\mathrm{k}_{\mathrm{B}}\right)$ and the absolute temperature $(\mathrm{T})$.

\section{F. Bandwidth limitations of the PM and CFM}

Two kinds of hopping experiments, PM and CFM [see Figs. 1(d) and $1(\mathrm{~g})]$, are presented in this work. In the PM, the trap position is kept constant and data acquisition is only limited by the sampling rate of the device, $1 \mathrm{kHz}$. All transitions occurring in a time scale below $1 \mathrm{~ms}$ cannot be followed by the instrument. This sampling rate is large enough to measure molecular transitions that occur in the $0.1-1$ second scale (e.g., at $25^{\circ} \mathrm{C}$ ). In the CFM, the force is kept constant using a feedback to keep constant the distance between the center of the optical trap and the center of the pipette bead. The feedback algorithm works by moving the piezoelectric actuators at a rate of $1 \mathrm{kHz}$. A typical time lag of the piezoelectric actuators is around $1 \mathrm{~ms}$. Moreover, the algorithm has a delay related to the fact that feedback cannot discriminate whether a change in the force signal is due to thermal noise or to a real transition. This algorithm delay has been estimated from power spectral measurements of the trap position in the CFM and is around 5$10 \mathrm{~ms} .{ }^{19,29}$ Given these limitations, the CFM experiment will miss short time events. In consequence, a kinetic underestimation takes place at low forces for the unfolding process and at high forces for the folding process as previously reported. ${ }^{19}$ The effect leads to an overestimation of the free energy 
difference and the total molecular extension. The latter is the sum of the distances $x_{F-T S}$ and $x_{T S-U}$ obtained from Eqs. (1) and (2), $x_{F-T S}+x_{T S-U}=x_{F-U}$.

\section{RESULTS}

\section{A. Room temperature $\left(25^{\circ} \mathrm{C}\right)$}

To determine the molecular extension and energy difference, it is necessary to fit Eqs. (1) and (2) to the experimental data [Fig. 2(a)]. In Fig. 2(a) are shown the average kinetic rates for a minimum of 4 different molecules obtained from CFM and PM hopping experiments at room temperature. Blue full squares correspond to the folding transition rates obtained from CFM while the red full squares are the unfolding rates for the same experiment. The solid lines correspond to the fits at Eqs. (1) and (2) for the corresponding transition. In addition, blue and red empty circles correspond to the folding and unfolding kinetic rates for the PM experiment. Dashed lines are the corresponding fits to the kinetic rates (red-unfolding and blue-folding). In Fig. 2(a), we can see that the solid lines

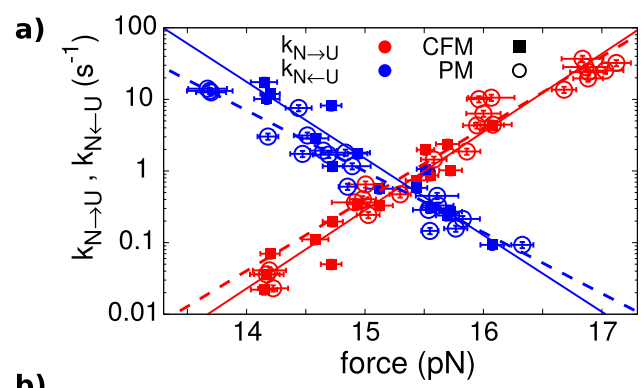

b)

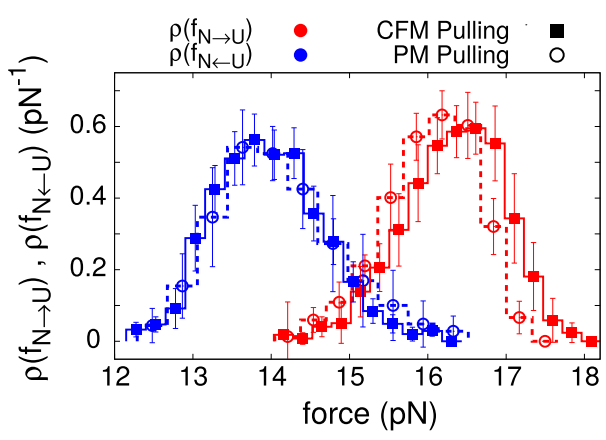

c)

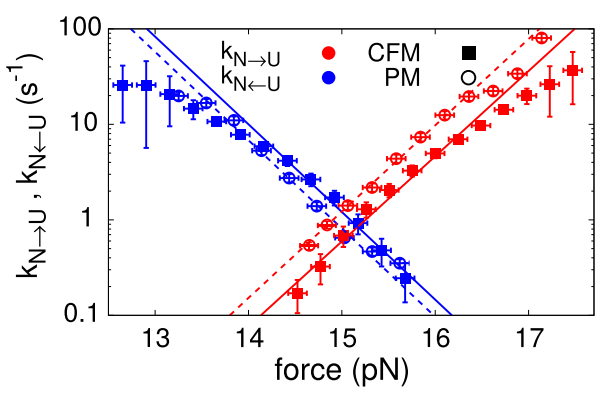

FIG. 2. (a) Unfolding kinetic rates (red dots) and folding kinetic rates (blue dots) for the PM (empty circles) and CFM (full squares) for hopping experiments at $25^{\circ} \mathrm{C}$. (b) Histograms of first rupture (red dots) and recovery force (blue dots) for PM (empty circles) and CFM (full squares) from pulling experiments at $25^{\circ} \mathrm{C}$. (c) Unfolding kinetic rates (red dots) and folding rates (blue dots) for PM (empty circles) and CFM (full squares) from pulling experiments at $25^{\circ} \mathrm{C}$. have a more pronounced slope as compared to the dashed ones, meaning larger distances $x_{F-T S}$ and $x_{T S-U}$ and a larger molecular extension $x_{F-U}$ in the CFM. Finally, we remark that in the studied force range, the behavior of kinetics from CFM and PM is well matched with the expected exponential force dependence so characteristic of the BE model. In Table I are summarized the obtained elastic and energetic parameters from both sets of data. There can be seen a difference in the molecular extension of $2.2 \pm 1.5 \mathrm{~nm}$ between the PM and CFM experiments. This difference agrees with reported values in previous studies with the same molecule. ${ }^{18,19}$ A difference of $12 \pm 2 \mathrm{k}_{\mathrm{B}} \mathrm{T}$ in $\Delta \mathrm{G}$ agrees with previous published results too. ${ }^{18}$

Pulling experiments in the PM and CFM have been also carried out to determine the kinetic rates of the designed DNA hairpin [Figs. 1(g)-1(i)]. Histograms of the first rupture and recovery forces have been measured with a minimum of 4 different molecules with at least 150 cycles per molecule [Fig. 2(b)]. PM experiments are done at $70 \mathrm{~nm} / \mathrm{s}$ and CFM experiments at an equivalent rate of $6 \mathrm{pN} / \mathrm{s}$. In Fig. 2(b), it is possible to see that the first rupture (red dots) and recovery (blue dots) forces match for pulling experiments in CFM (full squares) and PM (empty circles). We note that the mean rupture force is higher than the mean recovery force as expected. The results for the PM experiments are in agreement with the reported ones. ${ }^{22}$ However, the pulling experiments in the CFM are new results that have not been reported previously, to the best of our knowledge.

From those histograms are recovered the kinetic rates as a function of the force for both experiments [Fig. 2(c)]. In Fig. 2(c) are used the same color and symbol criteria than in Fig. 2(b) to denote the CFM and PM experiments and the folding or unfolding kinetic rates. We notice that the kinetic rates for the PM and CFM are compatible. The experimental results are fitted to Eqs. (1) and (2) to determine the molecular extension and energy difference. In Table I are summarized the more relevant parameters from those fits. Going in deep, we have recovered the following: (1) the kinetic rate at the coexistence force, which is close to the one obtained from hopping experiments $\left(0.9 \pm 0.2 \mathrm{~s}^{-1}\right.$ in pulling and $0.7 \pm 0.2 \mathrm{~s}^{-1}$ in hopping); (2) the sum of $x_{F-T S}$ and $x_{T S-U}$ yields the molecular extension with a difference between CFM and PM of only 0.1 $\pm 1.4 \mathrm{~nm}$; and (3) the total free energy difference between $\mathrm{F}$ and $\mathrm{U}$ is $0.6 \pm 1.9 \mathrm{k}_{\mathrm{B}} \mathrm{T}$ between both modes. Interestingly, the results of pulling experiments in the CFM do not overestimate distances and free energies as observed in CFM hopping experiments.

\section{B. Cold temperature $\left(6^{\circ} \mathrm{C}\right)$}

At low temperatures, there is much less thermal noise, the unzipping process exhibiting slower kinetics. In addition, lower temperatures contribute to preserve the molecular interaction between the beads and the handles thereby facilitating the experiments. In Fig. 3 are shown the kinetic results from hopping and pulling experiments with the same symbol and color code than at room temperature. Kinetic rate measurements from hopping experiments carried out in PM and CFM [Fig. 3(a)] are shown to be compatible. The lower the 
TABLE I. Results from the linear fits of the $\log (\mathrm{k})$ versus force for CFM and PM experiments at room and cold temperatures. ${ }^{\mathrm{a}, \mathrm{b}, \mathrm{c}}$

\begin{tabular}{lcccrrr}
\hline \hline \multicolumn{7}{c}{ Room temperature } \\
\hline & $\mathrm{f}_{\mathrm{c}}(\mathrm{pN})$ & $\mathrm{k}_{\mathrm{c}}\left(\mathrm{s}^{-1}\right)$ & \multicolumn{1}{c}{$\Delta \mathrm{G}\left(\mathrm{k}_{\mathrm{B}} \mathrm{T}\right)$} & \multicolumn{1}{c}{$\mathrm{x}_{\mathrm{F}-\mathrm{TS}}(\mathrm{nm})$} & $\mathrm{x}_{\mathrm{TS}-\mathrm{U}}(\mathrm{nm})$ & $\mathrm{x}_{\mathrm{F}-\mathrm{U}}(\mathrm{nm})$ \\
\hline Hop. CFM & $15.2 \pm 0.5$ & $0.7 \pm 0.2$ & $77 \pm 1.5$ & $10.3 \pm 0.5$ & $10.1 \pm 0.6$ & $20.5 \pm 1.1$ \\
Hop. PM & $15.2 \pm 0.3$ & $0.7 \pm 0.2$ & $65 \pm 1.2$ & $10.3 \pm 0.5$ & $8.0 \pm 0.5$ & $18.3 \pm 0.9$ \\
Pul. CFM & $15.2 \pm 0.3$ & $0.9 \pm 0.2$ & $63 \pm 1.3$ & $8.5 \pm 0.5$ & $8.7 \pm 0.6$ & $17.2 \pm 1.1$ \\
Pul. PM & $15.0 \pm 0.3$ & $1.0 \pm 0.2$ & $63 \pm 1.4$ & $8.6 \pm 0.5$ & $8.7 \pm 0.4$ & $17.3 \pm 0.6$ \\
\hline \multicolumn{7}{c}{ Cold temperature } \\
Hop. CFM & $17.7 \pm 0.3$ & $0.2 \pm 0.1$ & $84 \pm 1.5$ & $8.6 \pm 0.8$ & $9.8 \pm 0.6$ & $18.2 \pm 1$ \\
Hop. PM & $17.7 \pm 0.2$ & $0.2 \pm 0.1$ & $82 \pm 1.1$ & $10.2 \pm 0.6$ & $7.6 \pm 0.6$ & $17.8 \pm 0.8$ \\
Pul. CFM & $17.6 \pm 0.3$ & $0.3 \pm 0.1$ & $78 \pm 1.8$ & $9.1 \pm 0.6$ & $8.0 \pm 0.5$ & $17.1 \pm 1.1$ \\
Pul. PM & $17.5 \pm 0.3$ & $0.3 \pm 0.1$ & $76 \pm 1.6$ & $9.0 \pm 0.5$ & $7.7 \pm 0.6$ & $16.7 \pm 1.1$ \\
\hline \hline
\end{tabular}

${ }^{\mathrm{a}}$ The presented values are the mean \pm standard deviation over 4 different molecules.

${ }^{\mathrm{b}}$ The error for $\mathrm{k}_{\mathrm{c}}$ is obtained by propagating the errors in force and extension from Eqs. (1) and (2).

${ }^{\mathrm{c}} \mathrm{Hop}$. is hopping and Pul. is pulling.

temperature, the lower the strength of the thermal forces and the higher the value of the coexistence force, as expected. The

a)

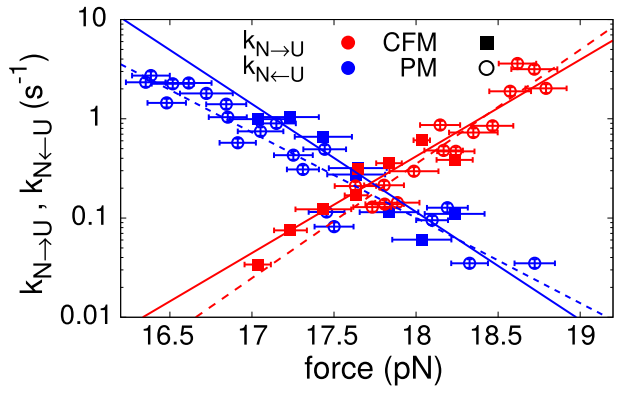

b)

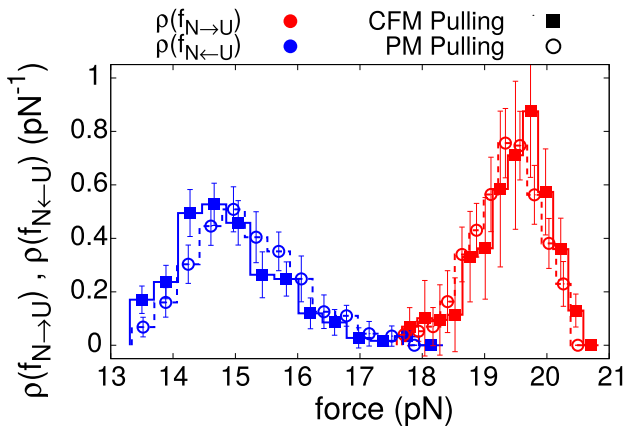

c)

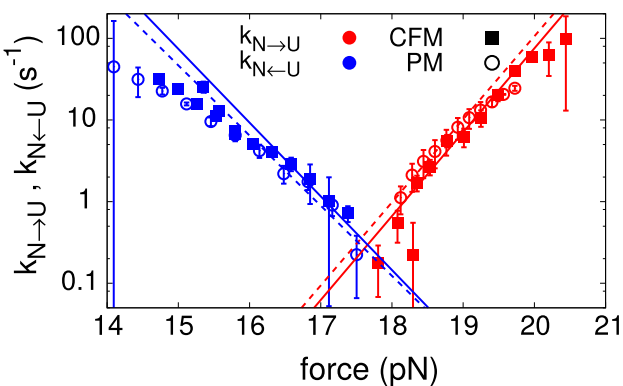

FIG. 3. (a) Unfolding kinetic rates (red dots) and folding kinetic rates (blue dots) for PM (empty circles) and CFM (full squares) from hopping experiments at $6{ }^{\circ} \mathrm{C}$. (b) Histograms of first rupture (red dots) and recovery force (blue dots) for PM (empty circles) and CFM (full squares) from pulling experiments at $6{ }^{\circ} \mathrm{C}$. (c) Unfolding kinetic rates (red dots) and folding rates (blue dots) for PM (empty circles) and CFM (full squares) from pulling experiments at $6{ }^{\circ} \mathrm{C}$. experimental parameters obtained from fitting data to Eqs. (1) and (2) are summarized in Table I. The values of the change in molecular extension $x_{F-U}$ and the free energy of formation $\Delta G$ measured in the CFM and PM are compatible within errors.

Pulling experiments at $6 \mathrm{pN} / \mathrm{s}(\mathrm{CFM})$ and $70 \mathrm{~nm} / \mathrm{s}(\mathrm{PM})$ have been also carried out. A minimum of 4 different molecules and 150 trajectories per molecule were collected. As we can see in Fig. 1(d), the hysteresis between the unfolding and folding trajectories increases with respect to room temperature measurements, Fig. 3(b). As shown in Fig. 3(c), the unfolding and folding rates obtained using the PM and CFM match perfectly throughout the force range. Let us note that while the coexistence force in pulling and hopping (in both the PM and CFM) is compatible with each other $(17.7 \pm 0.6 \mathrm{pN})$, the range of forces where kinetic rates are measured is much larger in the pulling case [see Figs. 3(a) and 3(c)]. A curvature in the force-dependent kinetic rates is apparent in Fig. 3(c) as predicted by the Leffler-Hammond postulate. ${ }^{26}$ The parameters fitting Eqs. (1) and (2) to the experimental data shown in Fig. 3(c) are shown in Table I. The values of the change in molecular extension $x_{F-U}$ and the free energy of formation $\Delta G$ measured in the CFM and PM are compatible within errors. They are also compatible to the values obtained from hopping experiments.

\section{Hot temperature $\left(45^{\circ} \mathrm{C}\right)$}

Hopping experiments at high temperatures show faster kinetic rates and lower coexistence forces corresponding to larger thermal forces [Fig. 1(d)]. We also expect, for CFM data, an increased fraction of missed transition events that should lead to a larger overestimation of both the change in molecular extension $x_{F-U}$ and the free energy of formation $\Delta G$ as compared to the room temperature case. The question remains whether such overestimation is also observed in pulling experiments, either in the PM or in the CFM.

In contrast to the cold case larger forces are required to unzip the DNA hairpin [Fig. 1(g)], a minimum of 5 different molecules with at least 100 pulling trajectories per molecule 
a)

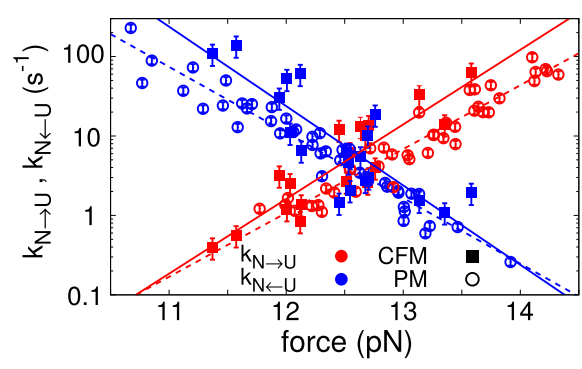

b)

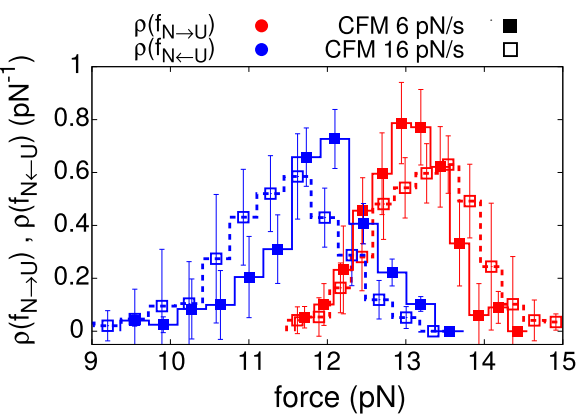

c)

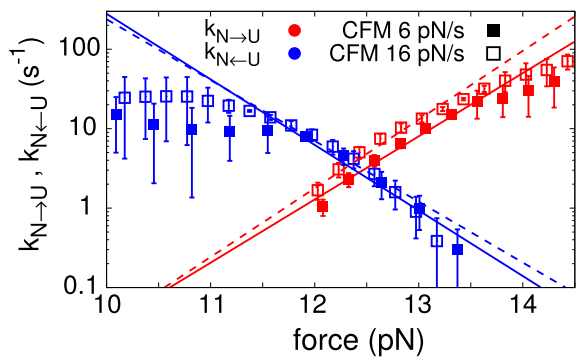

FIG. 4. (a) Unfolding kinetic rates (red dots) and folding kinetic rates (blue dots) for PM (empty circles) and CFM (full squares) from hopping experiments at $45{ }^{\circ} \mathrm{C}$. (b) Histograms of first rupture (red dots) and recovery force (blue dots) for the CFM pulling experiments at $6 \mathrm{pN} / \mathrm{s}$ (full squares) and 16 $\mathrm{pN} / \mathrm{s}$ (empty squares) at $45^{\circ} \mathrm{C}$. (c) Unfolding kinetic rates (red dots) and folding rates (blue dots) for the CFM pulling experiments at two different loading rates, $6 \mathrm{pN} / \mathrm{s}$ (full squares) and $16 \mathrm{pN} / \mathrm{s}$ (empty squares) at $45^{\circ} \mathrm{C}$.

have been studied. The kinetic measured from hopping experiments are shown in Fig. 4(a). In this plot, the CFM points are presented as full squares, red for the unfolding, and blue for the folding, while the points for the PM are denoted as empty circles with the same color criteria. As expected, the results obtained from CFM and PM differ due to missed transitions in the CFM. The overestimation of the molecular extension $x_{F-U}$ and free energy difference $\Delta \mathrm{G}$ are $3.2 \pm 1.3 \mathrm{~nm}$ and 12 $\pm 2.5 \mathrm{k}_{\mathrm{B}} \mathrm{T}$, respectively. All the parameters obtained from the fitting Eqs. (1) and (2) to the kinetic rates are summarized in Table II.

Pulling experiments were also carried out at two different loading and pulling rates in the CFM and PM. In CFM, the two loading rates are 6 and $16 \mathrm{pN} / \mathrm{s}$ [full and empty squares, respectively, in Figs. 4(b) and 4(c)]. In Fig. 4(b) are presented the histograms of the first rupture (red) and recovery (blue) force in the CFM. As expected, the difference between the mean rupture and mean recovery force increases when the speed is increased. This agrees with previous PM experiments of the same hairpin at room temperature. ${ }^{22}$ From those histograms, we have determined the kinetic rates in the CFM [Fig. 4(c)] that are compatible for both loading rates. Interestingly, the curvature in the $\log (\mathrm{k})$ vs. force plots is more apparent at this high temperature revealing a larger shift of the position of the transition state with force. ${ }^{26}$ The solid and dashed lines are fits of Eqs. (1) and (2) to the experimental data at $6 \mathrm{pN} / \mathrm{s}$ and $16 \mathrm{pN} / \mathrm{s}$, respectively. Fits were done around the coexistence force value where a linear relationship between $\log (\mathrm{k})$ and force is observed. In Table II are presented some of the obtained kinetic parameters of the fits. The values of the molecular extension $x_{F-U}$ and free energy difference $\Delta \mathrm{G}$ in the PM hopping experiments and the CFM pulling experiments are in good agreement. Therefore, no overestimation of relevant kinetic parameters from pulling experiments in the CFM is observed.

Pulling experiments in the PM were done at the equivalent pulling speeds of 70 and $225 \mathrm{~nm} / \mathrm{s}$. At both speeds, an unexpected result was found which is more striking at 70 $\mathrm{nm} / \mathrm{s}$. At this speed, the histograms along the force axis of the first rupture and recovery force distributions appear exchanged as if the dissipated work was negative (rather than positive), apparently against the second law [full circles in Fig. 5(a)]. Looking in detail to Fig. 1(g), we can see 5 different unfolding and folding trajectories where the molecule hops many times before it reaches the final state. The fact that there are many transitions along a single pulling curve means that the first rupture force is not indicative of the overall dissipation

TABLE II. Results from the linear fits of the $\log (\mathrm{k})$ versus force for CFM and PM at hot temperature. ${ }^{\mathrm{a}, \mathrm{b}, \mathrm{c}}$

\begin{tabular}{|c|c|c|c|c|c|c|}
\hline \multicolumn{7}{|c|}{ Hot temperature } \\
\hline & $\mathrm{f}_{\mathrm{c}}(\mathrm{pN})$ & $\mathrm{k}_{\mathrm{c}}\left(\mathrm{s}^{-1}\right)$ & $\Delta \mathrm{G}\left(\mathrm{k}_{\mathrm{B}} \mathrm{T}\right)$ & $\mathrm{x}_{\mathrm{F}-\mathrm{TS}}(\mathrm{nm})$ & $\mathrm{x}_{\mathrm{TS}-\mathrm{U}}(\mathrm{nm})$ & $\mathrm{x}_{\mathrm{F}-\mathrm{U}}(\mathrm{nm})$ \\
\hline Hop. CFM & $12.6 \pm 0.3$ & $6 \pm 2$ & $59 \pm 1.5$ & $9.5 \pm 0.5$ & $10 \pm 1$ & $19.6 \pm 1.1$ \\
\hline Hop. PM & $12.6 \pm 0.2$ & $5 \pm 2$ & $47 \pm 1.5$ & $8.1 \pm 0.5$ & $8.3 \pm 0.5$ & $16.4 \pm 0.7$ \\
\hline \multicolumn{7}{|c|}{ Pulling experiments } \\
\hline $6 \mathrm{pN} / \mathrm{s}$ & $12.4 \pm 0.3$ & $4 \pm 2$ & $52 \pm 2$ & $8.0 \pm 0.5$ & $8.3 \pm 0.6$ & $16.3 \pm 1.1$ \\
\hline $16 \mathrm{pN} / \mathrm{s}$ & $12.4 \pm 0.3$ & $4 \pm 1.9$ & $46 \pm 2$ & $8.7 \pm 0.8$ & $7.7 \pm 0.6$ & $16.4 \pm 1.1$ \\
\hline $70 \mathrm{~nm} / \mathrm{s}$ & $12.5 \pm 0.2$ & $31 \pm 10$ & $47 \pm 1.8$ & $8.1 \pm 0.5$ & $9.0 \pm 0.4$ & $17.0 \pm 0.7$ \\
\hline $225 \mathrm{~nm} / \mathrm{s}$ & $12.9 \pm 0.2$ & $16 \pm 7$ & $49 \pm 2$ & $8.0 \pm 0.5$ & $8.6 \pm 0.5$ & $16.6 \pm 0.7$ \\
\hline
\end{tabular}

${ }^{a}$ The presented values are the mean \pm standard deviation over 4 different molecules.

${ }^{\mathrm{b}}$ The error for $\mathrm{k}_{\mathrm{c}}$ is obtained by propagating the errors in force and extension from Eqs. (1) and (2).

${ }^{\mathrm{c}} \mathrm{Hop}$. is hopping and Pul. is pulling. 
a)
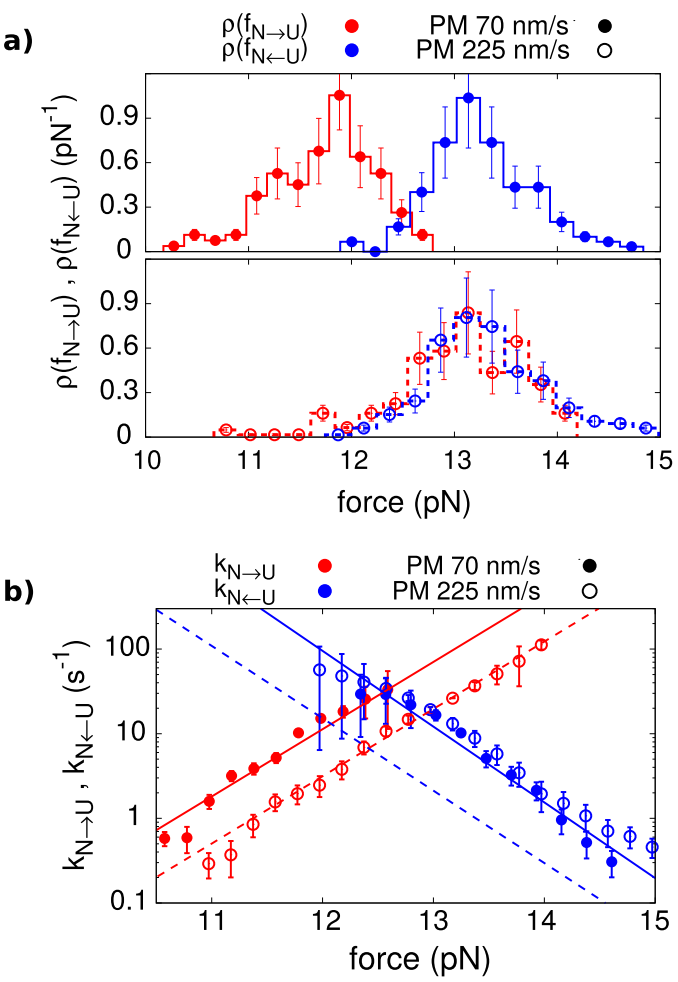

FIG. 5. (a) Histograms of first rupture (red dots) and recovery force (blue dots) for the pulling PM experiments at two pulling rates, $70 \mathrm{~nm} / \mathrm{s}$ (full circles, Top) and $225 \mathrm{~nm} / \mathrm{s}$ (empty circles, Bottom), at $45^{\circ} \mathrm{C}$. (b) Unfolding kinetic rates (red dots) and folding rates (blue dots) at $45^{\circ} \mathrm{C}$ for PM pulling experiments at $70 \mathrm{~nm} / \mathrm{s}$ (full circles) and $225 \mathrm{~nm} / \mathrm{s}$ (empty circles).

and that the average dissipated work also gets contributions from multiple unfolding-folding events. In fact, if the pulling speed is increased to $225 \mathrm{~nm} / \mathrm{s}$ (empty circles), both histograms overlap at the same force regime approaching the standard regime where first rupture forces are typically larger than first folding forces. Interestingly, the folding force distributions do not change with pulling speed, whereas the unfolding force distributions do. Kinetic rates are shown in Fig. 5(b) where a curvature for the kinetic rate vs force plots is not seen. The fitting parameters for both pulling speeds are summarized in Table II. The values of the measured molecular extension $x_{F-U}$ and free energy difference $\Delta \mathrm{G}$ agree with those reported from PM hopping experiments and CFM pulling experiments. Only hopping experiments in the CFM overestimate such values.

\section{DISCUSSION AND CONCLUSIONS}

Force spectroscopy studies are often done in two standard experimental modes, the constant force mode (CFM) and the passive mode (PM). In the CFM in optical tweezers or AFM, the force is controlled using force feedback. In the PM, the position of the force actuator (optical trap in optical tweezers, cantilever in AFM) is controlled; however, force fluctuates due to thermal noise or changes due to conformational transitions. Moreover, single-molecule experiments are often carried out in equilibrium conditions (hopping experiments) or outof-equilibrium (pulling experiments). Both operational modes (CFM and PM) and both kind of experiments (hopping and pulling) are suitable to investigate the kinetics of molecular folders at the single molecule level. In hopping experiments, the CFM has been traditionally preferred in order to directly measure force-dependent kinetic rates, whereas in pulling experiments, the PM is always used. The main advantage of the CFM in hopping experiments is the automated correction for drift effects in the force. In contrast, such effects are always present in the PM resulting in noisier traces and larger errors in kinetic rate measurements. Although the use of dual traps has much alleviated this problem, most optical tweezer setups still use the single trap configuration where force drift in the PM is unavoidable. In contrast, the main disadvantage of the CFM is the finite bandwidth of the feedback that introduces undesired noise effects and delays in the measurements that often lead to missed hopping transitions and overestimation of kinetic rates and free energy differences. ${ }^{19}$ In pulling experiments where the trap position (optical tweezers) or cantilever height (AFM) position is continuously changed and force varied between a minimum and a maximum value, force drift effects are not so acute as in hopping experiments, and the need of the CFM appears less justified. Therefore, pulling experiments in optical tweezers and AFM are always carried out in the PM.

In this paper, we have investigated the performance of the CFM and PM in both hopping and pulling experiments. Previous studies have shown limitations of the CFM in hopping experiments related to missing fast transitions due to the finite response time of the feedback. As shown in Ref. 19, this effect should therefore be more pronounced in systems with faster kinetic rates. However, it remains unclear whether such limitation is encountered in nonequilibrium pulling experiments in the CFM where irreversibility and dissipation effects effectively decrease the overall fraction of missed transitions.

In this paper, we have carried out force spectroscopy experiments on a DNA hairpin at three different temperatures: room temperature $\left(25^{\circ} \mathrm{C}\right)$, a cold temperature $\left(6^{\circ} \mathrm{C}\right)$, and a hot temperature $\left(45^{\circ} \mathrm{C}\right)$ measuring the unfolding and folding kinetic rates in hopping and pulling experiments in both CFM and PM. In general, we find that pulling experiments in both the CFM and PM recover the correct values of the kinetic rates and the corresponding parameters of the free energy landscape, such as molecular extension and free energy of formation. In contrast, hopping experiments yield the correct parameters only in the PM, whereas in the CFM, the values of the molecular extension of the hairpin and the free energy are overestimated due to missed transitions. ${ }^{19}$ This effect is noticeable at room temperature, negligible at low temperature (where hopping kinetics is slower) and dramatic at large temperature (where hopping is faster). Our results are summarized in Tables I and II. More specifically (1) the molecular extension at room temperature $\left(25^{\circ} \mathrm{C}\right)$ from CFM is $12 \%$ larger than the expected one from PM, which agrees with the results in Refs. 18 and 19. (2) At low temperature $\left(6^{\circ} \mathrm{C}\right)$, this difference is on the same order of magnitude of our experimental error. (3) The free energy of formation $\Delta \mathrm{G}$ measured at $25^{\circ} \mathrm{C}$ using the CFM is $18 \%$ higher than the measured one with PM. (4) The values of $\Delta \mathrm{G}$ measured at $6{ }^{\circ} \mathrm{C}$ using $\mathrm{CFM}$ an PM are compatible within the experimental errors. In addition, we carried out hopping experiments at $45^{\circ} \mathrm{C}$ and confirmed that for high 
kinetic rates, higher than at room temperature, the feedback is inefficient with many missed transitions and large overestimation effects in the CFM (Table II): (1) the molecular extension measured from CFM is $20 \%$ larger than the one measured from PM experiments. (2) The $\Delta \mathrm{G}$ measured in CFM is $25 \%$ higher than in PM.

Moreover, pulling experiments in a DNA hairpin controlling the trap position (PM) and the force (CFM) have been done at the same temperatures. This is the first time that both kind of pulling experiments, CFM and PM, are done and compared in this temperature range. We have shown that pulling experiments in the CFM and PM yield the correct force-dependent kinetic rates at the three temperatures. The results are summarized in Tables I and II. From those results, we confirm that both kind of pulling modes, CFM and PM, give us compatible molecular extensions and free energy differences at each temperature. In addition, pulling experiments present advantages compared to hopping experiments: (1) A larger force range to extract the kinetic rates is covered in pulling experiments. (2) Pulling experiments are less influenced by force drift by aligning individual trajectories in the data analysis. (3) Irreversible pulling experiments result in a faster experimental protocol than equilibrium hopping experiments. (4) Data obtained from irreversible pulling experiments allow us to use other methods, e.g., the fluctuation theorem, to extract the free energy of formation of the hairpin. Moreover, we have verified the presence of a concave curvature in the $\log (\mathrm{k})$ versus force plots as predicted by the Leffler-Hammond postulate. ${ }^{26}$ This concavity is more visible in CFM than in PM data.

Interestingly a new effect has been observed in pulling experiments in the $\mathrm{PM}$ at $45{ }^{\circ} \mathrm{C}$ and slow pulling rates, $70 \mathrm{~nm} / \mathrm{s}$, where the mean first unfolding force is lower than mean first recovery force, the unfolding force distribution being shifted to the left of the recovery force distribution. This result may look as counter-intuitive and seemingly against the second law. However, it is not as the average dissipated work along the pulling cycle is still positive if one considers all hopping events during the pulling cycle. In Fig. 1(g), one observes the high number of hopping events at $45{ }^{\circ} \mathrm{C}$ along single pulling trajectories. The work done upon the hairpin (e.g., along the stretching part of the cycle) equals the area below the force-distance curve, yet this work gets contributions from the multiple transition events along the curve rather than just the first force jump. To confirm that this effect is only related to the low pulling speed, we have done experiments at $225 \mathrm{~nm} / \mathrm{s}$. At this velocity, we have observed that both histograms approach each other overlapping over the same range of forces. The "normal ordering" of force distributions is then recovered at larger pulling speeds. It is interesting to note that this reversal effect of the first unfolding and folding force distributions allows us to extract kinetic rates over a very broad range of forces, in particular it allows us to determine kinetic rates below the coexistence rate value.

Finally, we have found that in irreversible pulling experiments in the CFM, the effect of overestimating distances and energies is less critical than in hopping experiments. Although the feedback algorithm in pulling experiments suffers from the same finite bandwidth limitations as in hopping experiments, the fraction of missed transitions is decreased for the pulling case under irreversible conditions where the first unfolding and recovery transition event is stabilized by the fast increasing (unfolding) or decreasing (folding) force. This is only true in cases where the average first unfolding (folding) force is larger (smaller) than the coexistence force, i.e., under nonequilibrium or irreversible conditions where average dissipation is larger than $\mathrm{k}_{\mathrm{B}} \mathrm{T}$. Otherwise, if dissipation is low, the pulling protocol becomes equivalent to the hopping protocol rendering the CFM inefficient too.

Overall we have confirmed that the overestimation error committed in the determination of the molecular extension and $\triangle \mathrm{G}$ using the CFM in hopping experiments comes from missed transitions (19), and that this effect tends to increase with temperature as expected. In contrast, irreversible pulling experiments in the CFM lead to more reliable results. We conclude that irreversible pulling experiments are a valuable tool to determine the force-dependent kinetic rates in the temperature range $5{ }^{\circ} \mathrm{C}-50{ }^{\circ} \mathrm{C}$.

\section{ACKNOWLEDGMENTS}

All authors acknowledge Spanish Research Council Grant No. FIS2016-80458-P, European Union's Horizon 2020 Grant No. 687089, and ICREA Academia 2013.

${ }^{1}$ F. Ritort, J. Phys.: Condens. Matter 18, R531 (2006).

${ }^{2}$ K. C. Neuman and A. Nagy, Nat. Methods 5(6), 491 (2008),

${ }^{3}$ C. Gosse and V. Croquette, Biophys. J. 82(6), 3314 (2002).

${ }^{4}$ B. Cappella and G. Dietler, Surf. Sci. Rep. 34(1-3), 5 (1999).

${ }^{5}$ X. Ding, S. S. Lin, B. Kiraly, S. Li, I. Chiang, J. Shi, S. J. Benkovic, and T. J. Huang, Proc. Natl. Acad. Sci. U. S. A. 109(28), 11105 (2012).

${ }^{6}$ S. B. Smith, Y. Cui, and C. Bustamante, Methods Enzymol. 361, 134 (2003).

${ }^{7}$ S. de Lorenzo, M. Ribezzi-Crivellari, J. R. Arias-Gonzalez, S. B. Smith, and R. Ritort, Biophys. J. 108, 2854 (2015).

${ }^{8}$ S. B. Smith, L. Finzi, and C. Bustamante, Science 258, 1122 (1992).

${ }^{9}$ S. B. Smith, Y. Cui, and C. Bustamante, Science 271, 795 (1996).

${ }^{10}$ J. M. Huguet, C. V. Bizarro, N. Forns, S. B. Smith, C. Bustamante, and F. Ritort, Proc. Natl. Acad. Sci. U. S. A. 107(35), 15431 (2010).

${ }^{11}$ J. B. Liphardt, B. Onoa, S. B. Smith, I. Tinoco, Jr., and C. Bustamante, Science 292, 733 (2001).

${ }^{12}$ M. Manosas, J. D. Wen, P. T. X. Li, S. B. Smith, C. Bustamante, I. Tinoco, Jr., and F. Ritort, Biophys. J. 92, 3010 (2007).

${ }^{13}$ C. Cecconi, E. A. Shank, C. Bustamante, and S. Marqusee, Science 309, 2057 (2005)

${ }^{14}$ J. C. Gebhardt, T. Bornschlögl, and M. Rief, Proc. Natl. Acad. Sci. U. S. A. 107, 2013 (2010).

${ }^{15}$ K. Svoboda, C. F. Schmidt, B. J. Schnapp, and S. M. Block, Nature 365, 721 (1993).

${ }^{16}$ M. T. Woodside, P. C. Anthony, W. M. Behnke-Parks, K. Larizadeh, D. Herschlag, and S. M. Block, Science 314, 1001 (2006).

${ }^{17}$ U. Bockelmann, Ph. Thomen, B. Essevaz-Roulet, V. Viasnoff, and F. Heslot, Biophys. J. 82, 1537 (2002).

${ }^{18}$ N. Forns, S. De Lorenzo, M. Manosas, K. Hayashi, J. M. Huguet, and F. Ritort, Biophys. J. 100, 1765 (2011).

${ }^{19}$ P. J. Elms, J. D. Chodera, C. Bustamante, and S. Marqusee, Biophys. J. 103, 1490 (2012).

${ }^{20}$ J. Camunas-Soler, M. Ribezzi-Crivellari, and F. Ritort, Annu. Rev. Biophys. 45, 65 (2016).

${ }^{21}$ W. J. Greenleaf, M. T. Woodside, E. A. Abbondanzieri, and S. M. Block, Phys. Rev. Lett. 95, 208102 (2005).

${ }^{22}$ A. Mossa, M. Manosas, N. Forns, J. M. Huguet, and F. Ritort, J. Stat. Mech.: Theory Exp. 2009, P02060. 
${ }^{23}$ D. Collin, R. Ritort, C. Jarzynski, S. B. Smith, I. Tinoco, Jr., and C. Bustamante, Nature 437, 231 (2005).

${ }^{24} \mathrm{G}$. Bell, Science 200, 618 (1978)

${ }^{25}$ E. Evans, A. Leung, D. Hammer, and S. Simon, Proc. Natl. Acad. Sci. U. S. A. 98(7), 3784 (2001).

${ }^{26}$ A. Alemany and F. Ritort, J. Phys. Chem. Lett. 8, 895 (2017).
${ }^{27}$ M. J. Lang, C. L. Asbury, J. W. Shaevitz, and S. M. Block, Biophys. J. 83(1), 491 (2002)

${ }^{28}$ J. Der Wen, M. Manosasm P. T. X. Li, S. B. Smith, C. Bustamante, F. Ritort, and I. Tinoco, Jr., Biophys. J. 92,2996 (2007).

${ }^{29}$ K. Hayashi, S. de Lorenzo, M. Manosas, J. M. Huguet, and F. Ritort, Phys. Rev. X 2, 031012 (2012). 